\title{
Indicadores de saúde auditiva em Minas Gerais - um \\ estudo por macrorregião
}

\section{Hearing Health indicators in the State of Minas Gerais - a study by macroregion}

\author{
Fernanda Jorge Maciel', Gabriela Cintra Januário², Cibele Martins Alvarenga Henriques³, Carolina Campos \\ Esteves 3 , Marcone Alexandre da Silva², Sirley Alves da Silva Carvalho ${ }^{4}$, Stela Maris Aguiar Lemos ${ }^{4}$
}

\section{RESUMO}

Objetivo: Analisar a organização da Rede Estadual de Atenção à Saúde Auditiva em Minas Gerais, no ano de 2009, tendo como referência os indicadores de fluxo/atenção ao usuário, de gestão do sistema (metas) e configuração de procedimentos e equipes de referência. Métodos: Estudo de abordagem quantitativa, analítico transversal, que considerou cinco variáveis centrais e nove variáveis complementares, para compreender a organização da Rede nas macrorregiões de saúde. Os dados foram obtidos no Sistema de Informação Ambulatorial e por meio de relatórios da Secretaria de Estado de Saúde de Minas Gerais. Foi realizada análise descritiva das variáveis e, para verificar a correlação entre elas, utilizou-se o Coeficiente de Correlação de Pearson e o teste t de Student. Resultados: Observou-se, entre as macrorregiões, variação no comportamento dos indicadores analisados, sendo que alguns Serviços de Atenção à Saúde Auditiva (SASA) parecem assumir serviços de outras regiões. Em todo o Estado, o valor médio de sessões de terapia fonoaudiológica por paciente, com Aparelho de Amplificação Sonora Individual (AASI), nas macrorregiões, foi de 10. No entanto, a média de produção de terapia foi inferior à média de adaptação de AASI, na maioria das macrorregiões. A grande demanda de adaptação parece estar orientando a organização dos serviços, que tem privilegiado a adaptação e não o acompanhamento, por meio das sessões de terapia individual. Conclusão: Acredita-se que o maior desafio da Rede seja fortalecer a dimensão da longitudinalidade do cuidado, descentralizadamente, por meio, principalmente, do serviço de fonoaudiologia descentralizada, propiciando maior efetividade aos serviços.

Descritores: Fonoaudiologia; Indicadores Básicos de Saúde; Avaliação de Programas e Projetos de Saúde; Políticas Públicas; Indicadores de Qualidade em Assistência à Saúde; Audição

\begin{abstract}
Purpose: Analysis of the organization of the Hearing Health Care Network of the State of Minas Gerais in 2009 using as reference the user flow/care indicators, system management (targets), procedure configuration and reference teams. Methods: Study of quantitative and cross-sectional analytical approach, considering five core variables and nine supplementary variables to understand the organization of the Network in the health macro-regions. The data were obtained from the Outpatient Information System and through reports of the Secretary of Health of the State of Minas Gerais. A descriptive analysis of the variables was made and the Pearson correlation coefficient and Student $\mathrm{t}$ test were used to verify the correlation between them. Results: It was observed that among the macro-regions there was a variation in the behavior of the analyzed indicators, whereby some Hearing Health Care Units (HHCU) seem to assume the services of other regions. Statewide, the average value of sessions of speech therapy per patient with Hearing aid (HA) in the macro-regions was 10 . However, the average therapy production was lower than the average HA adaptation in most of the macro-regions. The great demand for adaptation seems to guide the organization of the services, which has favored the adaptation and not the follow-up, through personal therapy sessions. Conclusion: It is believed that the greatest challenge of the Hearing Health Care Network of the State of Minas Gerais is to strengthen the longitudinal dimension of care, mainly through a decentralized speech therapy service, in order to improve the efficiency of the services.
\end{abstract}

Keywords: Speech, Language and Hearing Science; Health Status Indicators; Program Evaluation; Public Policies; Quality Indicators, Health Care; Hearing

Trabalho realizado no Departamento de Fonoaudiologia, Universidade Federal de Minas Gerais - UFMG - Belo Horizonte (MG), Brasil, com apoio da FAPEMIG, Edital PPSUS 2009.

(1) Escola de Saúde Pública do Estado de Minas Gerais - ESP/MG - Belo Horizonte (MG), Brasil.

(2) Secretaria de Estado de Saúde de Minas Gerais - SES/MG - Belo Horizonte (MG), Brasil.

(3) Bolsista de Apoio Técnico de Pesquisa da FAPEMIG - Belo Horizonte (MG), Brasil.

(4) Universidade Federal de Minas Gerais - UFMG - Belo Horizonte (MG), Brasil.

Conflito de interesses: Não

Contribuição dos autores: $F J M$ concepção e projeto, análise e interpretação dos dados, redação do artigo, revisão crítica relevante do conteúdo intelectual, aprovação final da versão a ser publicada; $G C J$ concepção e projeto, análise e interpretação dos dados, redação do artigo, revisão crítica relevante do conteúdo intelectual, aprovação final da versão a ser publicada; $C M A H$ redação do artigo, revisão crítica relevante do conteúdo intelectual; $C C E$ concepção e projeto, análise e interpretação dos dados; $M A S$ concepção e projeto, análise e interpretação dos dados; SASC concepção e projeto, análise e interpretação dos dados, redação do artigo, revisão crítica relevante do conteúdo intelectual, aprovação final da versão a ser publicada; $S M A L$ concepção e projeto, análise e interpretação dos dados, redação do artigo, revisão crítica relevante do conteúdo intelectual, aprovação final da versão a ser publicada.

Endereço para correspondência: Fernanda Jorge Maciel. Av. Augusto de Lima, 2061, Barro Preto, Belo Horizonte (MG), CEP: $30190-002$.

E-mail: fernandajmaciel@gmail.com

Recebido em: 29/1/2013; Aceito em: 14/10/2013 


\section{INTRODUÇÃO}

Segundo a Organização Mundial de Saúde ${ }^{(1)}$, estima-se que 42 milhões de pessoas acima de 3 anos de idade possuam algum tipo de deficiência auditiva, de grau moderado a profundo e que $1,5 \%$ da população brasileira, ou seja, cerca de 2.250 .000 habitantes, apresentem alguma deficiência auditiva, que está em terceiro lugar entre todas as deficiências do país. Em Minas Gerais, de acordo com os dados de Censo $2000^{(2)}, 15 \%$ da população relatou possuir algum tipo de deficiência (física, visual, mental ou auditiva) e desse total, $4 \%$ apresentam perda auditiva.

A literatura internacional ${ }^{(3)}$ aponta que, da população mundial com idade abaixo de 15 anos, aproximadamente 62 milhões possuem algum tipo de perda auditiva permanente. Desse número, cerca de dois terços (41 milhões) vivem em países em desenvolvimento.

A Política Nacional de Saúde Auditiva, instituída pela Portaria Ministerial no 2.073, de 28 de Setembro de 2004, é um grande marco para a saúde no Brasil, uma vez que inicia as discussões acerca da atenção integral e integrada aos indivíduos com deficiência auditiva, usuários do Sistema Único de Saúde (SUS). Um dos objetivos da Política é desenvolver estratégias de promoção da qualidade de vida, educação, proteção, recuperação da saúde e controle de danos. Objetiva, também, promover a ampla cobertura no atendimento a indivíduos com deficiência auditiva no Brasil, garantindo a universalidade do acesso, a equidade, a integralidade e o controle social da saúde auditiva. Além disso, define critérios técnicos mínimos para o funcionamento e a avaliação dos Serviços de Atenção à Saúde Auditiva $^{(4)}$.

Pautada nessa política, a Secretaria de Estado de Saúde de Minas Gerais implantou a Rede de Atenção à Saúde Auditiva, hierarquizada e regionalizada, com atendimento integral ao indivíduo com deficiência auditiva, realizado pelos Serviços de Atenção à Saúde Auditiva e outros pontos de atenção: Fonoaudiologia Descentralizada (Secretaria Municipal de Saúde/Atenção primária), Junta de Saúde Auditiva Microrregional, Junta Reguladora da Saúde Auditiva, Serviços de Referência de Triagem Auditiva Neonatal e Serviços de Implante Coclear. Em 2009, havia 12 Serviços de Saúde Auditiva credenciados, sete de alta complexidade e cinco de média complexidade ${ }^{(5)}$.

Avaliar o momento de implantação dessa política é essencial para compreender os avanços e desafios da Rede, fornecendo subsídios para a gestão, de modo a favorecer atendimento de qualidade para um maior número de usuários do Sistema Único de Saúde - SUS ${ }^{(6-8)}$. Além disso, a avaliação deve ser vista como um instrumento de mudança, sendo essencial incorporá-la às rotinas nas instâncias de gestão do SUS ${ }^{(9)}$. Nesse sentido, são necessárias evidências que mostrem quais os padrões de práticas efetivos, a fim de justificar a aplicação dos recursos.

Pessoas com alterações auditivas comparecem aos serviços de saúde auditiva com o objetivo de melhorar sua função auditiva e, paralelamente, o seu desempenho comunicativo. Quando o tratamento é oferecido, como a adaptação do AASI e/ou o serviço de reabilitação, a efetividade desse tratamento precisa ser analisada por todos os ângulos ${ }^{(10,11)}$.

A avaliação da gestão do cuidado, considerando as suas diversas dimensões - desde a individual até a social - deve ser capaz de apontar, não apenas os resultados que se espera (indicadores "duros"), como também revelar os processos produzidos e criados em todas as dimensões do cuidado. Essa proposta de avaliação vai além da compreensão de estrutura, processos e resultados ${ }^{(12)}$, na medida em que revela a complexidade e heterogeneidade do seu "objeto", perpassando, em todas as suas dimensões, pela internalidade e externalidade ${ }^{(8)}$. Embora seja essencial compreender tais aspectos, do ponto de vista da gestão do SUS, monitorar e avaliar as mudanças propicia respostas imediatas aos gestores para a definição de estratégias de intervenção. A construção de um painel de indicadores de saúde que contemple as demandas, os recursos, processo, produtos e resultados é apontado também como forma de incorporar a avaliação na gestão em saúde ${ }^{(12)}$, além de outras estratégias como as que mesclam ensino, aprendizagem e trabalho, ou que se utilizam das abordagens quantitativa e qualitativa, na perspectiva da rede de atenção, centrada na movimentação do usuário entre os pontos de atenção ${ }^{(13)}$. Nesse caso, é mais vantajoso iniciar a avaliação pela abordagem quantitativa, tendo em vista a disponibilidade de dados nos sistemas de informação ${ }^{(13)}$.

$\mathrm{Na}$ literatura especializada em Audiologia ${ }^{(14)}$, tem-se estudado os benefícios e a satisfação do usuário em relação ao uso do Aparelho de Amplificação Sonora Individual (AASI), como meio de avaliar os resultados dessa intervenção. No entanto, apenas esse tipo de análise não é suficiente para a avaliação da qualidade dos serviços de saúde auditiva, sendo indispensável a análise do desempenho dos serviços de saúde, no que se refere à efetividade, acesso, eficiência, respeito ao direito, dentre outros aspectos ${ }^{(15)}$.

Diante da relevância epidemiológica da deficiência auditiva, do seu impacto na qualidade de vida da população e da recente organização dos Serviços de Saúde Auditiva, faz-se necessário caracterizar a Rede de Saúde Auditiva do Estado de Minas Gerais, com vistas aos aspectos relacionados à estrutura e aos resultados obtidos. Dessa forma, espera-se fornecer subsídios ao planejamento, monitoramento e avaliação do serviço atualmente oferecido.

O presente estudo teve como objetivo analisar a organização da Rede Estadual de Atenção à Saúde Auditiva em Minas Gerais no ano de 2009, tendo como referência os indicadores de fluxo/ atenção ao usuário, de gestão do sistema (metas) e configuração de procedimentos e equipes de referência.

\section{MÉTODOS}

Este estudo faz parte do projeto "Avaliação da implementação da rede de atenção à saúde auditiva: um estudo de caso 
das microrregiões de Sete Lagoas e Curvelo, Minas Gerais", financiado pela Fundação de Amparo à Pesquisa do Estado de Minas Gerais (FAPEMIG) e aprovado pelo Comitê de Ética em Pesquisa da Universidade Federal de Minas Gerais (UFMG) (ETIC 186-10).

Trata-se de pesquisa quantitativa, retrospectiva e transversal, de natureza analítica, na qual se verifica o comportamento das variáveis selecionadas, no período de janeiro a dezembro de 2009.

A unidade de análise adotada neste trabalho foi a macrorregião de saúde, de acordo com o Plano Diretor de Regionalização de Minas Gerais (PDR) ${ }^{(16)}$, ainda que a Rede de Saúde Auditiva (RESA-MG) possua particularidades quanto à organização regional dos serviços de saúde no território (Figura 1). A escolha metodológica foi feita por permitir maior comparabilidade deste estudo, já que o PDR é parte da diretriz organizativa da regionalização do Sistema Único de Saúde ${ }^{(17)}$.

A Rede de Saúde Auditiva da SES-MG contava, em 2009, com 12 Serviços de Saúde Auditiva credenciados, habilitados pelo Ministério da Saúde, distribuídos no Estado de acordo com o Plano Diretor de Regionalização (Figura 1).

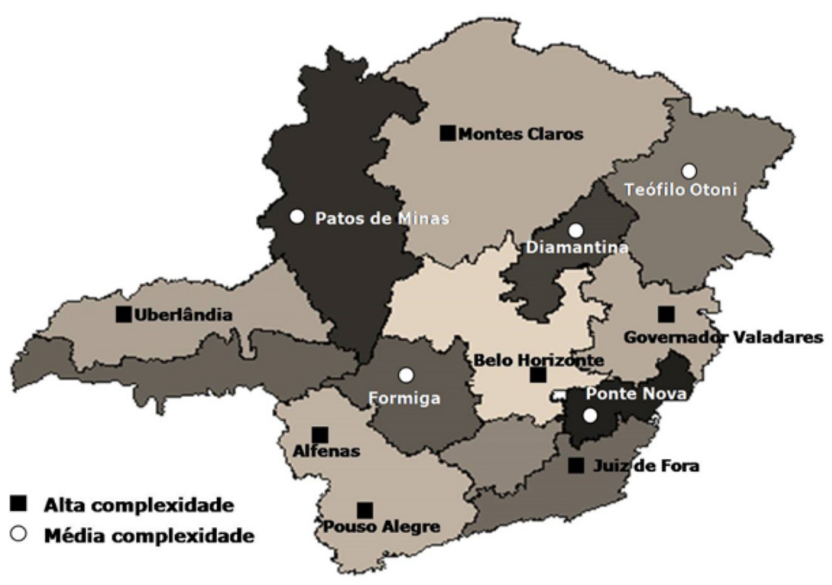

Fonte: Secretaria de Estado de Saúde de Minas Gerais

Figura 1. Municípios sede dos Serviços de Atenção à Saúde Auditiva

Os serviços de saúde auditiva são constituídos por Unidades de Média Complexidade e Alta Complexidade, seguindo o fluxo de referência e contrarreferência. (Figura 2). Os serviços de alta e média complexidade são responsáveis por receber os usuários agendados pelas Secretarias Municipais de Saúde, realizar avaliações médicas, otorrinolaringológicas, exames audiométricos, seleção e adaptação de Aparelho de Amplificação Sonora Individual (AASI), reabilitações/terapias fonoaudiológicas, acompanhamentos e reposições. Além disso, são responsáveis por realizar ações na Atenção Básica e oferecer suporte técnico para os Fonoaudiólogos Descentralizados. Ao Estado, cabe o controle e avaliação periódicos das unidades credenciadas para o serviço, além de promover a capacitação dos profissionais da rede e oferecer suporte, sempre que necessário ${ }^{(18)}$. A Deliberação CIB-SUS/MG no 464, de 17 de julho de $2008^{(19)}$, estabelece critérios para a definição de metas físicas de atendimento em média e alta complexidade da Rede Estadual de Saúde Auditiva para o Estado de Minas Gerais. No Art. $2^{\circ}$, fica definido que os serviços de atenção à saúde auditiva credenciados em alta complexidade deverão formalizar Plano Operativo, em que $70 \%$ dos procedimentos contratados serão de média complexidade em saúde auditiva e $30 \%$, de alta complexidade em saúde auditiva.

Dos 12 Serviços de Atenção à Saúde Auditiva (SASA) existentes em 11 macrorregiões de saúde, em 2009, cinco eram de média complexidade e sete de alta complexidade. Ressalta-se que os dois serviços de alta complexidade da região Sul foram analisados em conjunto e que as duas macrorregiões que ainda não possuíam SASA implantado (Centro Sul e Triângulo do Sul) encaminhavam os pacientes para as macrorregiões Sudeste e Triângulo do Norte, respectivamente.

\section{Fluxo do usuário na Rede de Atenção à Saúde Auditiva de Minas Gerais}

A RESA-MG possui diversos pontos de atenção, organizados de forma hierarquizada e regionalizada. A porta de entrada é o Serviço de Fonoaudiologia Descentralizada (FD), ou Referência Técnica em Saúde Auditiva do município de origem, excepcionalmente para os neonatos, cujo primeiro contato é na Rede no Serviço de Triagem Auditiva Neonatal (SRTAN). O FD realiza o acolhimento do usuário, referenciando-o, se necessário, para uma avaliação audiológica básica, preferencialmente realizada na Junta de Saúde Auditiva Microrregional. Se esse usuário for classificado como candidato ao uso do Aparelho de Amplificação Sonora Individual - AASI, a Junta Microrregional encaminha para a Junta Reguladora de Saúde Auditiva, que providencia o agendamento no Serviço de Atenção à Saúde Auditiva (SASA) de referência, conforme as prioridades clínicas da fila de espera. No SASA, são realizadas consultas com equipe interdisciplinar, exames complementares, seleção e adaptação de AASI e encaminhamento para cirurgia de Implante Coclear, quando necessário. Em seguida, o usuário é contrarreferenciado ao Fonoaudiólogo Descentralizado, que o acompanhará por meio das sessões de terapia fonoaudiológica individual e/ou monitoramento auditivo (Figura 2). A Fonoaudiologia Descentralizada, segundo a deliberação CIBSUS/MG nº 485, de 19 de novembro de 2008, tem como objetivo acolher e reabilitar os usuários da Rede de Saúde Auditiva, em seu município ou em municípios referenciados (Figura 2).

Cinco variáveis explicativas orientaram a descrição da Rede, denominadas aqui como variáveis centrais, por estarem diretamente relacionadas ao fluxo dos pacientes entre os principais pontos de atenção, funcionando, portanto, como indicadores. Outras nove variáveis, denominadas complementares, relacionadas a esses indicadores, foram analisadas de modo a contribuir para a melhor compreensão e descrição do funcionamento da Rede. Para cálculo dessas variáveis, foram 


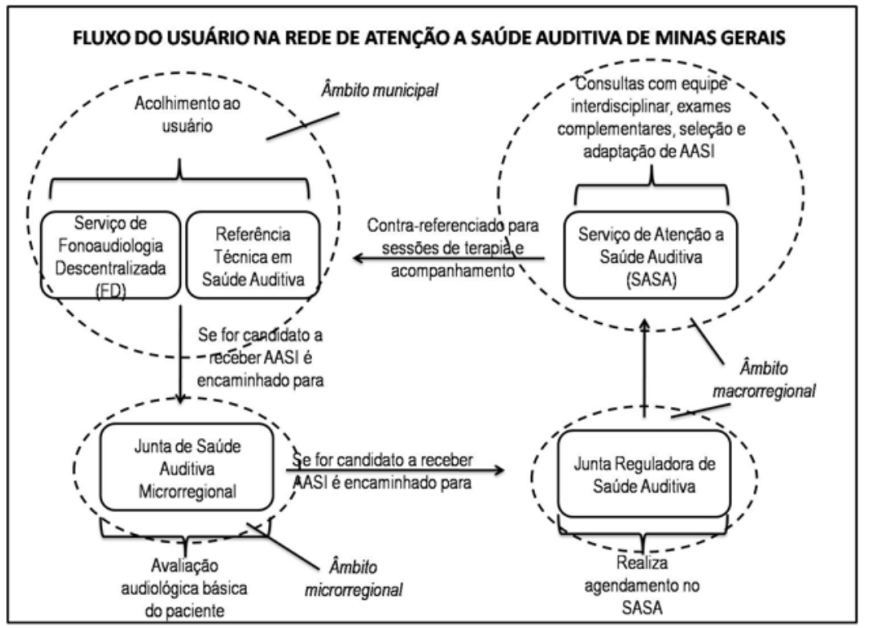

Figura 2. Fluxo do usuário na Rede de Atenção à Saúde Auditiva em Minas Gerais

considerados os procedimentos registrados e disponíveis no Sistema de Informação Ambulatorial (SIA) (Quadro 1). Vale ressaltar que o SRTAN não foi analisado por não possuir os dados disponíveis no Sistema de Informação Ambulatorial (SIA), uma vez que o Programa de Triagem Auditiva Neonatal em Minas Gerais é custeado apenas com recurso do tesouro estadual, não sendo esse procedimento registrado, portanto, nesse banco de dados nacional.

Os dados foram compilados em planilha Excel 2007 e analisados pelo software de análise estatística SPSS, versão 16. Para a análise descritiva, foram utilizados os valores mínimo e máximo, média e desvio padrão nas macrorregiões de saúde, além do valor absoluto observado em Minas Gerais. Foi utilizado o Coeficiente de Correlação de Pearson para verificar a correlação entre variáveis e o teste t de Student, considerando intervalo de confiança de 95\% e valor de significância de 5\%.

\section{RESULTADOS}

Inicialmente, procedendo à análise das variáveis que descrevem a configuração dos serviços e equipes de referência da Rede, nota-se que, nas macrorregiões de saúde, existiam, em média, $71,8 \%$ das microrregiões com Junta de Saúde Auditiva

Quadro 1. Descrição das variáveis centrais e complementares e centrais utilizadas no estudo

\begin{tabular}{|c|c|c|c|}
\hline Variáveis & Definição & Fórmula de cálculo e fonte & $\begin{array}{l}\text { Cálculo considerando os códigos } \\
\text { dos procedimentos no sistema de } \\
\text { informação ambulatorial }\end{array}$ \\
\hline \multicolumn{4}{|l|}{ Variáveis centrais } \\
\hline $\begin{array}{l}\text { Terapia fonoaudiológica } \\
\text { individual }\end{array}$ & $\begin{array}{l}\mathrm{N}^{\circ} \text { de sessões de terapia fonoaudiológica } \\
\text { individual realizadas. Essas sessões são } \\
\text { indicadas após a adaptação do AASI e } \\
\text { devem ser realizadas preferencialmente } \\
\text { com o fonoaudiólogo descentralizado. No } \\
\text { entanto, também devem ser realizadas no } \\
\text { SASA no início do processo terapêutico. }\end{array}$ & $\begin{array}{l}\mathrm{n}^{\circ} \text { de sessões realizadas } \\
\text { Fonte: SIA/DATASUS }\end{array}$ & $=030107011-3$ \\
\hline $\begin{array}{l}\text { Aparelhos de Amplificação } \\
\text { Sonora Individual (AASI) }\end{array}$ & $\begin{array}{l}\mathrm{N}^{\circ} \text { de aparelhos adaptados nos pacientes. } \\
\text { A adaptação ocorre no SASA de referência } \\
\text { de cada macrorregião de saúde. }\end{array}$ & $\begin{array}{l}\mathrm{n}^{\circ} \text { de AASI adaptados } \\
\text { Fonte: SIA/DATASUS }\end{array}$ & $\begin{array}{c}=(070103012-7+070103013-5 \\
+070103014-3+070103003-8 \\
+070103004-6+070103005-4 \\
+070103006-2+070103007-0 \\
+070103008-9+070103009-7 \\
+070103010-0+070103011-9+ \\
070103001-1+070103002-0)\end{array}$ \\
\hline
\end{tabular}


Quadro 1. Descrição das variáveis centrais e complementares e centrais utilizadas no estudo (cont.)

\begin{tabular}{|c|c|c|c|}
\hline Variáveis & Definição & Fórmula de cálculo e fonte & $\begin{array}{c}\text { Cálculo considerando os códigos } \\
\text { dos procedimentos no sistema de } \\
\text { informação ambulatorial }\end{array}$ \\
\hline \multicolumn{4}{|l|}{ Variáveis complementares } \\
\hline $\begin{array}{l}N^{\circ} \text { de sessões de terapia } \\
\text { fonoaudiológica individual por } \\
\text { paciente adaptado }\end{array}$ & $\begin{array}{l}\mathrm{N}^{\circ} \text { de sessões de terapia fonoaudiológica } \\
\text { individual realizadas por paciente que } \\
\text { recebeu o AASI. }\end{array}$ & $\begin{array}{l}\text { [n }{ }^{\circ} \text { sessões de fonoterapia/ } \\
\left(\mathrm{n}^{\circ} \text { pacientes }\right){ }^{*} O \mathrm{n}^{\circ} \text { de } \\
\text { pacientes adaptados foi } \\
\text { calculado considerando que } \\
\text { cada paciente recebe em } \\
\text { média } 2 \text { AASI. } \\
\text { Fonte: SIA/DATASUS }\end{array}$ & $\begin{array}{c}=[(030107011-3) /((070103012-7 \\
+070103013-5+070103014-3 \\
+070103003-8+070103004-6 \\
+070103005-4+070103006-2+ \\
070103007-0+070103008-9+ \\
070103009-7+070103010-0+ \\
070103011-9+070103001-1+ \\
070103002-) / 2)]\end{array}$ \\
\hline $\begin{array}{l}\text { Índice de Efetividade da } \\
\text { Avaliação audiológica básica }\end{array}$ & $\begin{array}{l}\text { Índice criado pelos pesquisadores } \\
\text { que reflete a efetividade da avaliação } \\
\text { audiológica básica no que se refere } \\
\text { ao fluxo dos pacientes entre os pontos } \\
\text { de atenção. Para isso, considerou-se } \\
\text { que essa avaliação deve ser capaz } \\
\text { de classificar aqueles usuários com } \\
\text { deficiência auditiva e que tenham perfil } \\
\text { para recebimento do AASI e que, por } \\
\text { isso, devam ser encaminhados para } \\
\text { uma avaliação detalhada no SASA de } \\
\text { referência. }\end{array}$ & $\begin{array}{l}\text { [n }{ }^{\circ} \text { deavaliações audiológicas } \\
\text { para diagnóstico para } \\
\text { diagnósticos de deficiência } \\
\text { auditiva / }\left(n^{\circ} \text { de pacientes }\right. \\
\left.\text { adaptados }{ }^{*}\right) \text { ] } \\
\text { Fonte: SIA/DATASUS }\end{array}$ & $\begin{array}{c}=[(021107009-2+021107010-6) \\
/((070103012-7+070103013-5 \\
+070103014-3+070103003-8+ \\
070103004-6+070103005-4+ \\
070103006-2+070103007-0+ \\
070103008-9+070103009-7+ \\
070103010-0+070103011-9+ \\
070103001-1+070103002-) / 2)]\end{array}$ \\
\hline $\begin{array}{l}\text { \% de JSAM de referência para } \\
\text { o SASA, oficializadas. }\end{array}$ & $\begin{array}{l}\text { Refere-se ao número de JSAM } \\
\text { oficializadas em sua macrorregião } \\
\text { em relação ao total de microrregiões } \\
\text { referenciadas naquela macrorregião. }\end{array}$ & $\begin{array}{l}\text { [n }{ }^{\circ} \text { de JSAM oficializadas } \\
/ \mathrm{n}^{\circ} \text { de microrregiões } \\
\text { referenciadas] } \\
\text { Fonte: SES/MG }\end{array}$ & Não se aplica \\
\hline $\begin{array}{l}\% \text { de FD que encaminham para } \\
\text { o SASA, oficializadas. }\end{array}$ & $\begin{array}{l}\text { Refere-se ao número de fonoaudiólogos } \\
\text { descentralizados oficializados em um } \\
\text { determinado município em relação ao } \\
\text { total de municípios referenciados na } \\
\text { macrorregião. }\end{array}$ & $\begin{array}{l}\text { [n }{ }^{\circ} \text { de FD oficializados } / \mathrm{n}^{\circ} \\
\text { de municípios referenciados] } \\
\text { Fonte: SES/MG }\end{array}$ & Não se aplica \\
\hline Carga horária & $\begin{array}{l}\text { Refere-se à carga horária semanal em } \\
\text { horas dos profissionais que atuam no } \\
\text { SASA de média e de alta complexidade. } \\
\text { Essa carga horária foi auto referida pelos } \\
\text { próprios profissionais. Correspondente } \\
\text { a quatro variáveis: carga horária do } \\
\text { Otorrinolaringologista; do Fonoaudiólogo; } \\
\text { do Psicólogo e do Assistente Social. }\end{array}$ & $\begin{array}{l}\mathrm{n}^{\circ} \text { de horas por semana } \\
\text { Fonte: SES/MG }\end{array}$ & Não se aplica \\
\hline Audiometria Tonal Limiar & $\begin{array}{l}\text { Número de exames de audiometria tonal } \\
\text { limiar realizados. Esses exames podem } \\
\text { ocorrer no município com Fonoaudiologia } \\
\text { Descentralizada, na JMSA ou em outros } \\
\text { serviços de saúde credenciados. Esse } \\
\text { exame pode ser contabilizado também } \\
\text { dentre as avaliações audiológicas no } \\
\text { SASA. }\end{array}$ & $\begin{array}{l}\mathrm{n}^{\circ} \text { de exames realizados } \\
\text { Fonte: SIA/DATASUS }\end{array}$ & $=021107004-1$ \\
\hline
\end{tabular}

Legenda: SASA = Serviço de Atenção à Saúde Auditiva; AASI = Aparelho de Amplificação Sonora Individual; SIA = Sistema de Informação Ambulatorial; JMSA = Junta Microrregional de Saúde Auditiva; SES = Secretaria de Estado de Saúde de Minas Gerais; JMSA = Junta Microrregional de Saúde Auditiva

e 38,2\% dos municípios com fonoaudiólogos descentralizados oficializados (Tabela 1).

No que se refere à gestão do sistema, a Rede Estadual contava, em 2009, com 13.800 metas de média e alta complexidade, que possibilitariam a entrada de aproximadamente 0,7 novos pacientes a cada 1.000 habitantes, em todo o Estado. Observou-se, no entanto, que esse número variava de forma importante entre as macrorregiões, podendo chegar a 2,8 novos pacientes/1.000 habitantes na região do Jequitinhonha, cujo município polo é Diamantina.

Ao se comparar o número de metas com o número de novos pacientes recebidos, constatou-se que a Rede teria capacidade de atender mais 2.969 novos pacientes no ano analisado. Por meio do teste t de Student, verificou-se que essa diferença, nas macrorregiões, é estatisticamente significativa $(\mathrm{t}=-3,797$, $\mathrm{p}=0,04 ; \mathrm{IC}=111,5 ; 428,3)$. 
Tabela 1. Medidas resumo das variáveis quantitativas analisadas no estudo em Minas Gerais e nas macrorregiões de saúde no ano de 2009

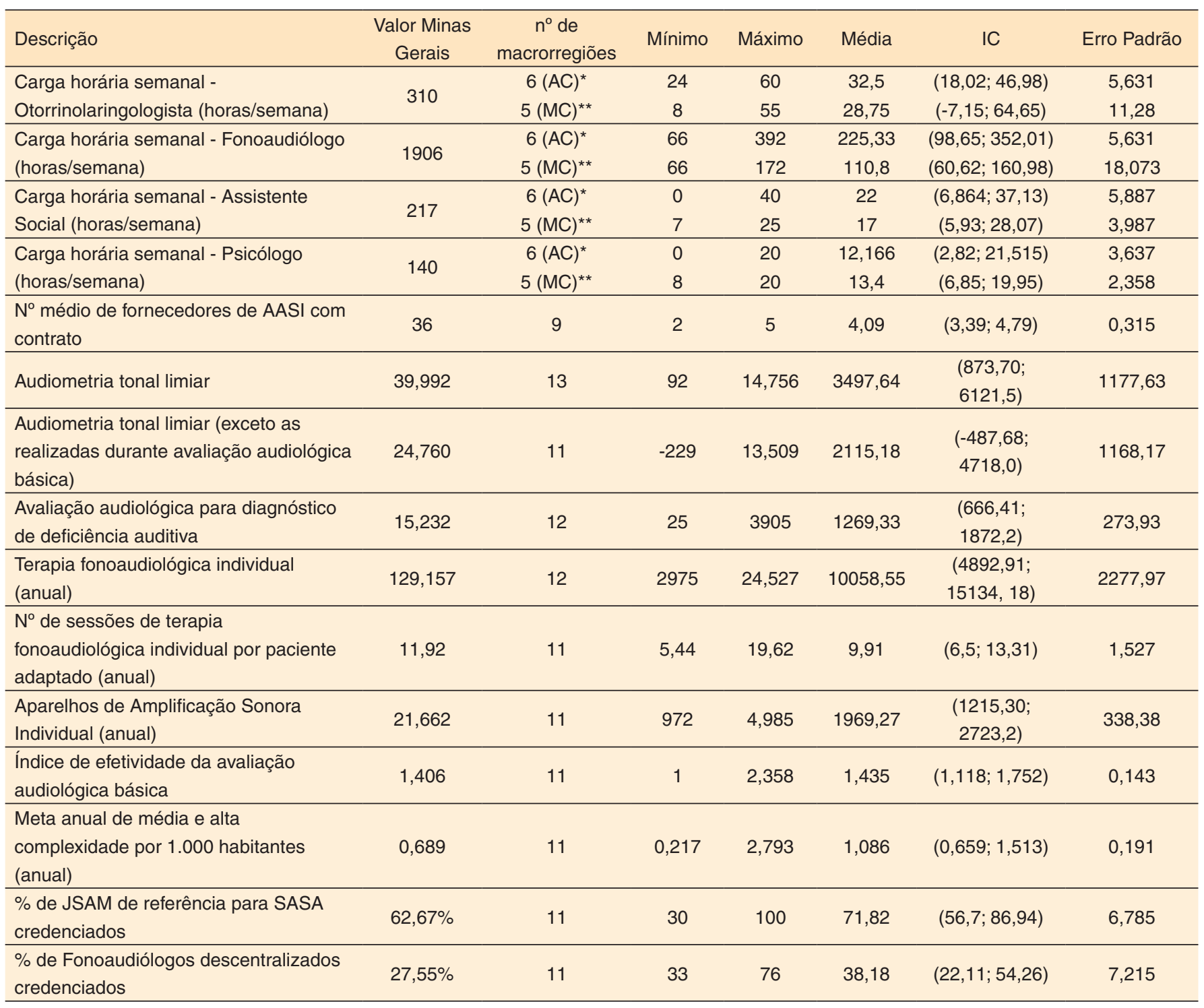

Fonte: DATASUS e Secretaria de Estado de Saúde de Minas Gerais

* Serviço de Atenção à Saúde Auditiva de média complexidade; ** Serviço de Atenção à Saúde Auditiva de alta complexidade

*** Considerou-se as macrorregiões que possuíam informações referentes a cada uma das variáveis. Além disso, no ano de 2009 apenas as 11 macrorregiões que possuíam o Serviço de Atenção à Saúde auditiva

Legenda: $\mathrm{AC}=$ alta complexidade, $\mathrm{MC}=$ média complexidade

Considerando os indicadores de fluxo/atenção ao usuário, notou-se que as macrorregiões Jequitinhonha, Leste do Sul, Noroeste, Norte e Oeste tinham maior semelhança no comportamento das seguintes variáveis: metas de média e alta complexidade, avaliação diagnóstica de deficiência auditiva e número de pacientes que receberam aparelho auditivo. Em relação às variáveis avaliação diagnóstica e adaptação de AASI, que compõem o índice de efetividade da avaliação audiológica básica, observou-se que nas macrorregiões Leste, Nordeste, Sul e Triângulo do Norte o número de avaliações apresentou-se maior que o número de pacientes que receberam aparelho, em comparação com as demais macrorregiões (Figura 3).

Em relação ao número de avaliações de pacientes, as macrorregiões Leste, Nordeste, Sudeste e Sul tiveram número superior, onde também o número de pacientes avaliados apresentou diferença importante, em relação ao número de pacientes adaptados. A maior diferença entre pacientes avaliados e pacientes com aparelho foi encontrada na macrorregião Sul. Esse mesmo comportamento pôde ser observado entre as variáveis de avaliação audiológica para diagnóstico de deficiência auditiva, número de AASI adaptados e número de metas de média e alta complexidade (Figura 3) nas macrorregiões de saúde e na correlação estatisticamente significativa encontrada (Tabela 2).

Ressalta-se que na macrorregião Sul havia dois SASA de alta complexidade, esperando-se, assim, que a produção total dessa macrorregião fosse o dobro das outras macrorregiões que tinham apenas uma SASA de alta complexidade.

Ainda com relação à atenção ao usuário da Rede, foram 


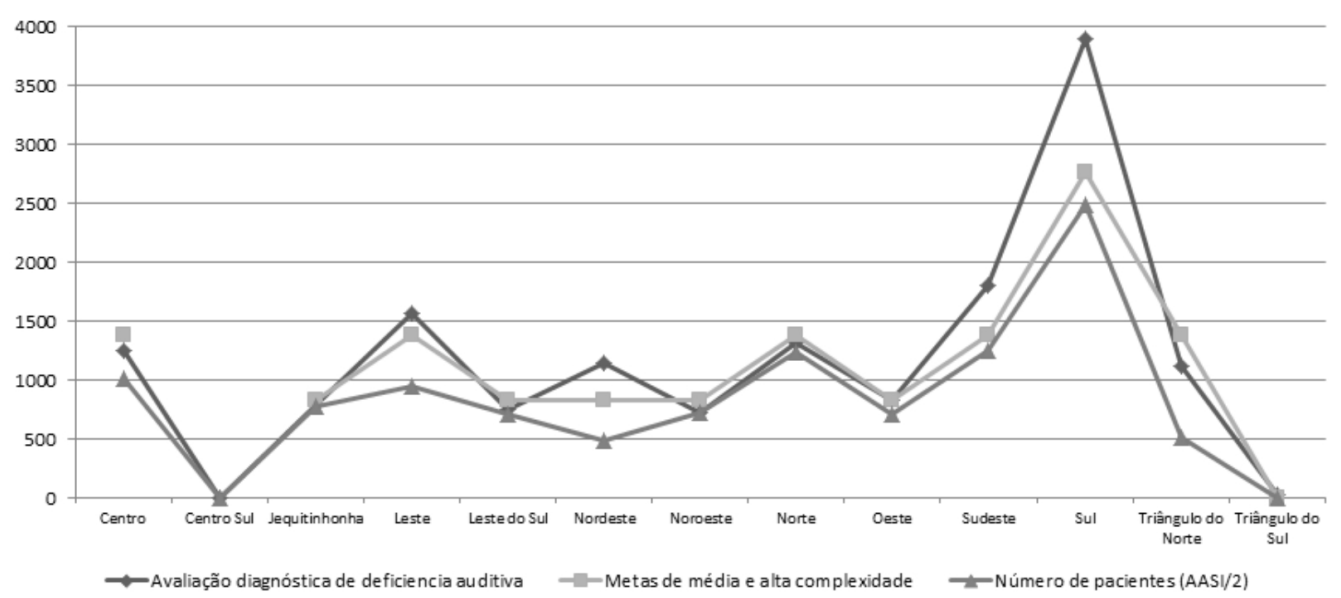

Legenda: AASI = Aparelho de Amplificação Sonora Individual; AASI/2 = optou-se por dividir o número de adaptações por dois, uma vez que mais de $80 \%$ dos pacientes são adaptados bilateralmente segundo dados fornecidos pela Secretaria Estadual de Saúde de Minas Gerais

Figura 3. Comportamento das variáveis metas de média e alta complexidade, avaliação diagnóstica de deficiência auditiva e aparelhos de amplificação sonora individual nas macrorregiões de saúde de Minas Gerais em 2009

Tabela 2. Relação entre as variáveis metas de média e alta complexidade, avaliação diagnóstica de deficiência auditiva e aparelhos de amplificação sonora individual nas macrorregiões de saúde de Minas Gerais em 2009

\begin{tabular}{lcccc}
\hline \multirow{2}{*}{ Descrição } & \multicolumn{2}{c}{ Avaliação diagnóstica de deficiência auditiva } & \multicolumn{2}{c}{ Metas de média e alta complexidade } \\
\cline { 2 - 5 } & $\mathrm{r}$ & valor de $\mathrm{p}$ & $\mathrm{r}$ & valor de $\mathrm{p}$ \\
\hline Aparelhos de Amplificação Sonora Individual & 0,936 & 0,000 & 0,913 & 0,000 \\
Metas de média e alta complexidade & 0,936 & 0,000 & - & - \\
\hline
\end{tabular}

Coeficiente de correlação de Pearson $(r)(p<0,05)$

realizados 24.760 exames de audiometria tonal limiar em Minas Gerais, voltados exclusivamente para a detecção de deficiência auditiva e identificação da necessidade de adaptação de AASI, com posterior encaminhamento para o SASA de referência (Tabela 1). Para verificar a efetividade da classificação dos pacientes como candidatos a receber a adaptação após a realização da avaliação audiológica básica, foi criado o índice de efetividade da avaliação audiológica básica (Quadro 1), que deve estar o mais próximo do valor unitário, uma vez que o esperado é que todos os usuários que realizaram a avaliação audiológica completa no SASA de fato tenham perfil para o recebimento do AASI. O Índice em Minas Gerais e seu valor médio nas macrorregiões atingiram valores próximos a 1,4, estando em torno de $40 \%$ acima do ideal (Tabela 1). Além disso, não foi possível identificar correlação estatisticamente significativa entre as variáveis de avaliação audiológica para diagnóstico de deficiência auditiva e AASI (coeficiente de correlação de Pearson de $-0,3$ e p=0,931).

Em todo o Estado, foram realizadas, aproximadamente, 12 sessões de terapia fonoaudiológica individual para cada paciente adaptado e o valor médio das macrorregiões foi de aproximadamente dez sessões, chegando até 19 na macrorregião Sudeste (Tabela 1). Para verificar se a reabilitação auditiva vinha ocorrendo de forma descentralizada nos municípios, ou se ainda estão ocorriam principalmente nos SASA, foi verificada a correlação entre a carga horária do fonoaudiólogo no Serviço de Atenção à Saúde Auditiva (ou
SASA) e o número de sessões de terapia fonoaudiológica individual. Foi encontrada, de forma estatisticamente significativa, uma forte correlação (coeficiente de correlação de Pearson de 0,884 e p=0,000) entre essas duas variáveis, uma vez que a variação do número médio mensal de sessões de fonoterapia e o número de AASI distribuídos ocorreram de forma concomitante na maioria das regiões (Figura 4). Isso sugere que a fonoterapia, possivelmente, ainda esteja ocorrendo nos polos da macrorregiões e não de forma descentralizada, como previsto no desenho da Rede.

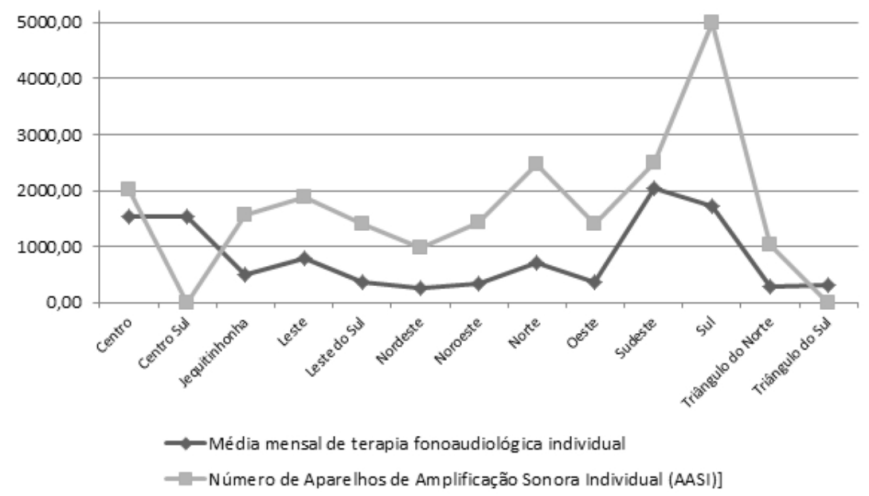

Legenda: $\mathrm{AASI}=$ Aparelho de Amplificação Sonora Individual

Figura 4. Distribuição do número médio mensal de sessões de terapia fonoaudiológica individual realizadas e número absoluto de Aparelhos de Amplificação Sonora Individual adaptados, nas macrorregiões de saúde de Minas Gerais no ano de 2009 
Quanto à adaptação de aparelhos, observou-se elevado número na macrorregião Sul, em relação às demais macrorregiões do Estado. No entanto, a média de produção de terapia fonoaudiológica não acompanhou a média de adaptação de AASI. Verificou-se que, nas macrorregiões onde não havia SASA (Centro Sul e Triângulo do Sul), havia produção de terapia fonoaudiológica.

\section{DISCUSSÃO}

A análise da organização da Rede Estadual de Atenção à Saúde Auditiva em Minas Gerais, no ano de 2009, mostrou variação no comportamento dos indicadores analisados, a saber: fluxo/atenção ao usuário, gestão do sistema (metas) e configuração de procedimentos e equipes de referência, em relação aos Serviços de Saúde Auditiva credenciados.

$\mathrm{O}$ fato de a macrorregião sul ter apresentado maior diferença entre o número de avaliações diagnósticas em relação ao número de metas de média e alta complexidade, pode estar baseado no Art. $4^{\circ}$ da deliberação CIB-SUS/MG no $464^{(19)}$, que prevê a revisão das metas/cotas físicas para os serviços de atenção à saúde auditiva credenciados em média e alta complexidade, quando necessário, conforme conveniência operacional e logística. Porém, esse procedimento de assumir serviços de outras regiões pode ser indicativo de deficiência na estratégia utilizada para se chegar à integralidade do atendimento, ou de que essa diferença seja decorrente da falta de infraestrutura para atender toda a demanda de adaptação de aparelhos auditivos.

Embora este estudo tenha constatado correlação significativa entre metas de média e alta complexidade, avaliação diagnóstica de deficiência auditiva e adaptação de aparelhos de amplificação sonora individual nas macrorregiões de saúde de Minas Gerais em 2009, o processo de reabilitação auditiva apresentou-se aquém do esperado, em relação ao número de indivíduos adaptados com AASI. Sabe-se que o processo de reabilitação da deficiência auditiva envolve um trabalho muito mais amplo, que prevê a adaptação de AASI de fluxo contínuo, com acompanhamento médico e fonoaudiológico, tanto para ajustes como para verificações periódicas das condições técnicas e do benefício obtido com o uso desses equipamentos, além de assistência social e psicológica ${ }^{(20)}$.

Atender o paciente apenas no momento da aquisição do aparelho não é suficiente. A falta do acompanhamento traz consequências, pois o uso não efetivo do aparelho auditivo compromete a integração social ${ }^{(21,22)}$. Sendo assim, a realização de terapias fonoaudiológicas para acompanhamento dos pacientes adaptados com AASI é fundamental, visando a integração do paciente no processo de comunicação, no convívio social, nas dimensões psicológica e profissional.

Dentre os profissionais envolvidos no processo de seleção e adaptação de um AASI estão os Otorrinolaringologistas, responsáveis, principalmente, pelo diagnóstico, e os fonoaudiólogos, cujo papel principal é a habilitação ou reabilitação do indivíduo com deficiência auditiva ${ }^{(23)}$. Para a American SpeechLanguage Hearing Association (ASHA) ${ }^{(24)}$ o fonoaudiólogo é responsável por reabilitar a função social da audição, uma vez que esse profissional aborda a utilidade prática da capacidade auditiva, visando aumentar a habilidade do indivíduo com deficiência auditiva em lidar com as situações da vida diária. Tais afirmações podem explicar o fato da carga horária semanal do Fonoaudiólogo ter sido superior a dos demais profissionais (Otorrinolaringologista, Psicólogo e Assistente Social), no presente estudo. Assim, o fonoaudiólogo torna-se responsável por grande parte da gestão do cuidado ao usuário dos serviços de Saúde Auditiva, bem como pela proposição de programas de reabilitação que visem a inclusão social dos deficientes auditivos $^{(24,25)}$.

A Portaria Ministerial n 2.073, de 2004, SAS n587 Anexo $I^{(4)}$, refere que as ações de Saúde Auditiva devem ocorrer da Atenção Básica aos serviços de Alta Complexidade, compreendendo ações de promoção à saúde auditiva, de prevenção e identificação precoce de problemas auditivos junto à comunidade, assim como ações informativas e educativas, orientação familiar e encaminhamentos, quando necessário, para o Serviço de Atenção à Saúde Auditiva.

Esse mesmo documento define o conjunto de atividades que a equipe deve desenvolver em Serviço de Atenção à Saúde Auditiva na Média Complexidade: triagem e monitoramento da audição de neonatos, pré-escolares e escolares, diagnóstico, tratamento e reabilitação de perda auditiva em crianças a partir de 3 anos de idade, de jovens, de adultos, incluindo os trabalhadores, e de idosos, respeitando as especificidades da avaliação e reabilitação exigidas para cada um desses segmentos, além de qualificar a assistência e promover a educação continuada dos profissionais de saúde envolvidos com a implantação e a implementação da Política de Atenção à Saúde Auditiva, em acordo com os princípios da integralidade e da humanização ${ }^{(4)}$.

No processo de seleção e de adaptação do aparelho de amplificação sonora individual, o sucesso da adaptação está relacionado ao trabalho de orientação e de aconselhamento. No entanto, o profissional precisa conhecer as reais dificuldades dos pacientes em relação ao uso adequado da amplificação ${ }^{(26)}$. Desse modo, as sessões de terapia permitirão avaliações e desenvolvimentos de estratégias para a utilização efetiva do AASI.

No estudo, pôde-se observar que a média de produção de terapia é bastante inferior à média de adaptação de AASI, salvo em duas das macrorregiões: Centro Sul e Triângulo do Sul que realizavam terapias fonoaudiológicas mesmo sem a existência do SASA. Vale ressaltar que o serviço de referência em saúde auditiva, na macrorregião Sul, superou o número de adaptações de AASI, chegando a média mensal 5000. Este número corresponde a $100 \%$ a mais que na segunda região com maior distribuição, a macrorregião Sudeste. Em contrapartida, a média mensal de terapia fonoaudiológica individual da macrorregião Sul não chegou a 2000. Algumas hipóteses podem ser levantadas para explicar esse resultado, dentre elas: a grande demanda 
de adaptação faz com que os serviços privilegiem a entrega e não o seguimento; a distância entre a moradia do paciente e o serviço faz com que o acesso à terapia seja dificultado; a cobertura para terapia está sendo negligenciada e não há contratação suficiente de fonoaudiólogos descentralizados nos municípios de moradia dos pacientes para garantir a terapia, não ocorrendo de forma descentralizada nas regiões.

Além dessa variação do número de sessões de fonoterapia entre as macrorregiões e ainda a sua possível realização de forma concentrada nos SASA, um estudo aponta a necessidade de se compreender os fatores relacionados ao comportamento desse processo de reabilitação na Rede. Isso porque a adaptação do AASI, essencial para que o indivíduo com deficiência auditiva desenvolva todo o seu potencial, prescinde da reabilitação para minimizar dificuldades relacionadas à perda auditiva. A terapia fonoaudiológica objetiva, desse modo, favorecer o uso efetivo do aparelho de amplificação sonora e individual e de estratégias que facilitem o desempenho do indivíduo na comunicação, com o máximo aproveitamento possível em cada contexto acústico ${ }^{(27)}$. A literatura aponta, ainda, que o treinamento auditivo possui efeitos positivos na adaptação de AASI ${ }^{27,28)}$, o que corrobora a importância dessa etapa do cuidado no fluxo da Rede.

Quanto à macrorregião Sul, que possui dois SASA, uma hipótese para o grande número de adaptações seria o acesso facilitado ao usuário, ou seja, não há barreiras que o impeçam de chegar ao serviço e há facilidade no diagnóstico, estimulando a entrada do usuário nesses serviços. Já na região de maior concentração de população, existe apenas um SASA. Nesse caso, vale lembrar a literatura ${ }^{(29)}$, que revela que quando o usuário encontra barreiras que impeçam sua entrada a um serviço da rede pública, ele encontra fluxos alternativos para chegar ao atendimento.

Os serviços de saúde da rede pública devem estabelecer diretrizes para o desenvolvimento de programas de diagnóstico, aquisição e adaptação/fornecimento de aparelhos de amplificação sonora individual e, principalmente, de reeducação auditiva para os deficientes auditivos, para que eles possam participar e desfrutar das relações sociais, mantendo uma boa qualidade de vida ${ }^{(29,30)}$. Cabe ressaltar, ainda, que o presente estudo mostrou avanços e desafios dos indicadores da rede de cuidado do usuário de AASI em um Estado que conta com a implantação da política de saúde auditiva em todo seu território. Apesar das contribuições do estudo, foram observadas algumas limitações, tais como o manejo de dados secundários, que não permitiu o aprofundamento na análise dos achados e o período de realização, muito próximo à implantação da política na SES-MG, 2009.

\section{CONCLUSÃO}

No presente estudo, discutiu-se a organização da rede de saúde auditiva em Minas Gerais, tendo como referência fluxos assistenciais, gestão do sistema e ordenação de procedimentos. Pode ser considerado relevante, pois contribui para o avanço da investigação na área.

Acredita-se que o maior desafio da Rede de Atenção à Saúde Auditiva em Minas Gerais seja fortalecer a dimensão da longitudinalidade do cuidado, que deve ocorrer descentralizadamente, de modo a propiciar maior eficiência do serviço e contribuir mais efetivamente para a melhoria da qualidade de vida da população com alteração auditiva. As evidências elencadas no presente estudo remetem também à necessidade de buscar estratégias de acompanhamento e avaliação das políticas públicas, considerando sistemas de informações em saúde como importantes instrumentos de gestão e planejamento.

\section{REFERÊNCIAS}

1. World Health Organization. Deafness and hearing impairment, 2006. [Internet] [citado 2010 Dez 13] Disponível em: http://www.who.int/ mediacentre/factsheets/fs300/en/index.html.

2. Instituto Brasileiro de Geografia e Estatística (IBGE). Censo Demográfico 2000: características da população e dos domicílios: resultados do universo. [Internet] [citado 2010 Dez 13]. Disponível em: http://www.ibge.gov.br/home/estatistica/populacao/censo2000/

3. Olusanya BO, Luxon LM, Wirz SL. Detection of permanent childhood hearing loss in a developing country. Nig Q J Hosp Med. 2011;21(4):324-6.

4. Brasil. Ministério da Saúde. Portaria GM/MS nº. 2.073, de 28 de setembro de 2004. Institui a Política Nacional de Atenção à Saúde Auditiva. [Internet] [citado 2010 Dez 13]. Disponível em: http:// dtr2001.saude.gov.br/sas/PORTARIAS/Port2004/GM/GM-2073.htm.

5. Armigliato ME, Prado DGA, Melo TM, Martinez MANS, Lopes AC, Amantini RCB, et al. Avaliação de serviços de saúde auditiva sob a perspectiva do usuário: proposta de instrumento. Rev Soc Bras Fonoaudiol. 2010;15(1):32-9.

6. Bosi MLM, Uchimura KY. Avaliação da qualidade ou avaliação qualitativa do cuidado em saúde? Rev Saúde Pública. 2007;41(1):150-3.

7. Cecílio LCO. Apontamentos teóricos-conceituais sobre processos avaliativos considerando as múltiplas dimensões da gestão do cuidado. Interface (Botucatu). 2011;15(37):589-99.

8. Carvalho ALB, Senra IMVB, Oliveira KC, Tanaka OU, Felisberto E, Alves CKA, et al. Práticas de monitoramento e avaliação: reflexões e resultados de um processo de cooperação interfederativo. Revista Tempus Actas de Saúde Coletiv. 2011;5(4):167-82.

9. Saunders GH, Chisolm TH, Abrams HB. Measuring hearing aid outcomes - not as easy as it seems. J Rehabil Res Dev. 2005;42(2):157-68.

10. Cook JA, Hawkins DB. Outcome measurement for patients receiving hearing aid services. Laryngoscope. 2007;117(4):610-3.

11. Tamaki EM, Tanaka OY, Felisberto E, Alves CKA, Drumond Junior M, Bezerra LCA, et al. Metodologia de construção de um painel de indicadores para o monitoramento e avaliação da gestão do SUS. Ciênc Saúde Colet. 2012;17(4):839-49. 
12. Tamaki EM, Barbieri AR, Cazola LHO, Andrade SMO, Tanaka OY. A incorporação da avaliação da atenção básica no nível estadual do Sistema Único de Saúde através de processos participativos de ensino-aprendizagem-trabalho. Rev Bras Saúde Mater Infant. Recife. 2012;10(Supl1):201-8.

13. Tanaka OY. Avaliação da atenção básica em saúde: uma nova proposta. Saúde Soc. 2011;20(4):927-34.

14. Bevilacqua MC, Melo TM, Morettin M, Lopes AC. A avaliação de serviços em Audiologia: concepções e perspectivas. Rev Soc Bras Fonoaudiol. 2009;14(3):421-6.

15. Viacava F, Ugá MAD, Porto S, Laguardia J, Moreira RS. Avaliação de desempenho de sistemas de saúde: um modelo de análise. Ciênc Saúde Colet. 2012;14(4):921-34.

16. Malachias I, Leles FAG, Pinto MAS. Plano Diretor de Regionalização da Saúde de Minas Gerais. Belo Horizonte: Secretaria de Estado de Saúde de Minas Gerais, 2010. [Internet] [citado 2013 out 26]. Disponível em: http://www.saude.mg.gov. br/images/documentos/Livro\%20Plano\%20Diretor\%20de $\% 20$ Regionalizacao\%20-\%20ultima\%20versao.pdf

17. Brasil. Ministério da Saúde. Lei $n^{\circ}$. 8.080, de 19 de Setembro de 1990. Dispõe sobre as condições para a promoção, proteção e recuperação da saúde, a organização e o funcionamento dos serviços correspondentes e dá outras providências. Brasília; 1990. [Internet] [citado 2011 Mar 25]. Disponível em: http://portal.saude.gov.br/ portal/arquivos/pdf/lei8080.pdf.

18. Agência Minas. Notícia do Governo do Estado de Minas Gerais. Saúde. Ações de mobilização alertam para os impactos do ruído sonoro. Belo Horizonte; 2011. [citado 2011 Jun]. Disponível em: http://www.agenciaminas.mg.gov.br/multimidia/galerias/acoes-demobilizacao-alertam-para-os-impactos-do-ruido-sonoro-2/.

19. Minas Gerais. Secretaria de Estado de Minas Gerais. Anexo único da Deliberação CIB-SUS/MG No 464, de 17 de julho de 2008 [Internet]. [citado 2008 Jul 25]. Disponível em: http://www.saude.mg.gov.br/ images/documentos/Anexo\%20Unico\%20da\%20De1\%20464\%20 Estabelece $\% 20$ criterios $\% 20$ meta\%20fisica.pdf.
20. Freitas CD, Costa MJ. Processo de adaptação de próteses auditivas em usuários atendidos em uma instituição pública federal - parte I: resultados e implicações com o uso da amplificação. Rev Bras Otorrinolaringol. 2007;73(6):744-51.

21. Veiga LR, Merlo ARC, Mengue SS. Satisfação com a prótese auditiva na vida diária em usuários do sistema de saúde do Exército Rev Bras Otorrinolaringol. 2005;71(1):67-73.

22. Teixeira CF. Estudo avaliativo da política de atenção à saúde auditiva: estudo de caso em Pernambuco - Recife [tese] Recife (PE): Centro de Pesquisas Ageu Magalhães, Fundação Oswaldo Cruz; 2007.

23. Zandavalli MB, Christmann LS, Garcez VRC. Rotina de procedimentos utilizados na seleção e adaptação de aparelhos de amplificação sonora individual em centros auditivos na cidade de Porto alegre, Brasil - RS. Rev CEFAC. 2009;11(1):106-15.

24. Ruschel CV, Carvalho CR, Guarinello AC. A eficiência de um programa de reabilitação audiológica em idosos com presbiacusia e seus familiares. Rev Soc Bras Fonoaudiol. 2007;12(2):95-8.

25. Campos K, Oliveira JRM, Blasca WQ. Processo de adaptação de aparelho de amplificação sonora individual: elaboração de um DVD para auxiliar a orientação a indivíduos idosos. Rev Soc Bras Fonoaudiol. 2010;15(1):19-25

26. Gil D. Treinamento auditivo formal em adultos com deficiência auditiva [tese]. São Paulo: Universidade de São Paulo; 2006.

27. Megale RL, Iório MCM, Schochat E. Treinamento auditivo: avaliação do benefício em idosos usuários de próteses auditivas. Pro Fono. 2010;22(2):101-6.

28. Tanaka OY, Drumond Junior M. Análise descritiva da utilização de serviços ambulatoriais no Sistema Único de Saúde segundo o porte do município, São Paulo, 2000 a 2007. Epidemiol Serv Saúde. 2010;19(4):355-66.

29. Veras RP, Mattos LC. Audiologia do envelhecimento: revisão da literatura e perspectivas atuais. Rev Bras Otorrinolaringol. 2007;73(1):128-34.

30. Gatto CL, Tochetto TM. Deficiência auditiva infantil: implicações e soluções. Ver CEFAC. 2007;9(1):110-5. 